\title{
Non-perturbative generation of elementary fermion mass: a numerical study
}

\section{S. Capitani ${ }^{a)}$, G.M. de Divitiis ${ }^{b)}$, P. Dimopoulos ${ }^{()}$, R. Frezzotti ${ }^{b) d)}$, M. Garofalo ${ }^{* d)}$, B.} Kostrzewa $^{e)}$, F. Pittlere) , G.C. Rossi ${ }^{(b)}$, C. Urbach ${ }^{e)}$

a) Johann Wolfgang Goethe-Universität Frankfurt am Main Institut für Theoretische Physik Max-von-Laue-Straße 1 D-60438 Frankfurt am Main Germany

b) Dipartimento di Fisica, Università di Roma "Tor Vergata"

c) Centro Fermi - Museo Storico della Fisica e Centro Studi e Ricerche Enrico Fermi, Compendio del Viminale, Piazza del Viminiale 1, I-00184, Rome, Italy

d) INFN, Sezione di Roma 2, Via della Ricerca Scientifica - 00133 Rome, Italy

e) Institut für Strahlen-und Kernphysik (Theorie), Nussallee 14-16 Bethe Center for Theoretical Physics, Nussallee 12 Universität Bonn, D-53115 Bonn, Germany

E-mail: marco.garofalodroma2.infn.it

In this talk we present a numerical lattice study of an SU(3) gauge model where an SU(2) doublet of non-Abelian strongly interacting fermions is coupled to a complex scalar field doublet via a Yukawa and a Wilson-like term. The model enjoys an exact symmetry, acting on all fields, which prevents UV power divergent fermion mass corrections, despite the presence of these two chiral breaking operators in the Lagrangian. In the phase where the scalar potential is non-degenerate and fermions are massless, the bare Yukawa coupling can be set at a critical value at which chiral fermion transformations become symmetries of the theory. Numerical simulations in the Nambu-Goldstone phase of the critical theory, for which the renormalized Yukawa coupling by construction vanishes, give evidence for non-perturbative generation of a UV finite fermion mass term in the effective action.

The 36th Annual International Symposium on Lattice Field Theory - LATTICE2018

22-28 July, 2018

Michigan State University, East Lansing, Michigan, USA.

${ }^{*}$ Speaker. 


\section{Introduction}

In [1] a new non-perturbative (NP) mechanism for elementary particle mass generation was conjectured. In this contribution we provide numerical evidence that this mass generation mechanism is realized in the simplest $d=4$ gauge model where this phenomenon can take place. The Lagrangian of the model is

$$
\begin{aligned}
& \mathscr{L}_{\text {toy }}(\Psi, A, \Phi)=\mathscr{L}_{\text {kin }}(\Psi, A, \Phi)+\mathscr{V}(\Phi)+\mathscr{L}_{\text {Wil }}(\Psi, A, \Phi)+\mathscr{L}_{\text {Yuk }}(\Psi, \Phi) \\
& \mathscr{L}_{\text {kin }}(\Psi, A, \Phi)=\frac{1}{4}(F \cdot F)+\bar{\Psi}_{L} \mathscr{D} \Psi_{L}+\bar{\Psi}_{R} \mathscr{D} \Psi_{R}+\frac{1}{2} \operatorname{tr}\left[\partial_{\mu} \Phi^{\dagger} \partial_{\mu} \Phi\right] \\
& \mathscr{V}(\Phi)=\frac{\mu_{0}^{2}}{2} \operatorname{tr}\left[\Phi^{\dagger} \Phi\right]+\frac{\lambda_{0}}{4}\left(\operatorname{tr}\left[\Phi^{\dagger} \Phi\right]\right)^{2} \\
& \mathscr{L}_{W i l}(\Psi, A, \Phi)=\frac{b^{2}}{2} \rho\left(\bar{\Psi}_{L} \overleftarrow{\mathscr{D}}_{\mu} \Phi \mathscr{D}_{\mu} \Psi_{R}+\bar{\Psi}_{R} \overleftarrow{\mathscr{D}}_{\mu} \Phi^{\dagger} \mathscr{D}_{\mu} \Psi_{L}\right) \\
& \mathscr{L}_{\text {Yuk }}(\Psi, \Phi)=\eta\left(\bar{\Psi}_{L} \Phi \Psi_{R}+\bar{\Psi}_{R} \Phi^{\dagger} \Psi_{L}\right),
\end{aligned}
$$

where $b^{-1}=\Lambda_{U V}$ is the UV-cutoff. The Lagrangian (1.1) describes a SU(2) fermion doublet subjected to non-Abelian gauge interaction and coupled to a complex scalar field via Wilsonlike (eq. (1.4)) and Yukawa (eq. (1.5)) terms. For short we use a compact SU(2)-like notation where $\Psi_{L}=\left(u_{L} d_{L}\right)^{T}$ and $\Psi_{R}=\left(u_{R} d_{R}\right)^{T}$ are fermion iso-doublets and $\Phi$ is a $2 \times 2$ matrix with $\Phi=\left(\phi,-i \tau^{2} \phi^{*}\right)$ and $\phi$ an iso-doublet of complex scalar fields. The term $\mathscr{V}(\Phi)$ in eq. (1.3) is the standard quartic scalar potential where the (bare) parameters $\lambda_{0}$ and $\mu_{0}^{2}$ control the self-interaction and the mass of the scalar field. In the equations above we have introduced the covariant derivatives

$$
\mathscr{D}_{\mu}=\partial_{\mu}-i g_{s} \lambda^{a} A_{\mu}^{a}, \quad \overleftarrow{\mathscr{D}}_{\mu}=\overleftarrow{\partial}_{\mu}+i g_{s} \lambda^{a} A_{\mu}^{a}
$$

where $A_{\mu}^{a}$ is the gluon field $\left(a=1,2, \ldots, N_{c}^{2}-1\right)$ with field strength $F_{\mu \nu}^{a}$, here $N_{c}=3$. Among other symmetries, the Lagrangian (1.1) is invariant under the global transformations $\left(\Omega_{L / R} \in \mathrm{SU}(2)\right)$

$$
\begin{aligned}
& \chi_{L} \times \chi_{R}=\left[\tilde{\chi}_{L} \times\left(\Phi \rightarrow \Omega_{L} \Phi\right)\right] \times\left[\tilde{\chi}_{R} \times\left(\Phi \rightarrow \Phi \Omega_{R}^{\dagger}\right)\right], \\
& \tilde{\chi}_{L / R}: Q_{L / R} \rightarrow \Omega_{L / R} Q_{L / R}, \quad \bar{Q}_{L / R} \rightarrow \bar{Q}_{L / R} \Omega_{L / R}^{\dagger} .
\end{aligned}
$$

No mass like term of the form $\bar{Q}_{L} Q_{R}+\bar{Q}_{R} Q_{L}$ can be generated as it is not $\chi_{L} \times \chi_{R}$ invariant.

The Lagrangian (1.1) is not invariant under the purely fermionic chiral transformations $\tilde{\chi}_{L} \times \tilde{\chi}_{R}$. However, in the phase with positive renormalized squared scalar mass $\left(\hat{\mu}_{\phi}^{2}>0\right)$, where the $\chi_{L} \times \chi_{R}$ symmetry is realized $a ̀ ~ l a$ Wigner, a critical value $\eta_{c r}$ of the bare Yukawa parameter exists where the effective Yukawa term can be made to vanish [1]. This critical value can be identified looking at the renormalized Schwinger-Dyson equation (SDE) of the Axial $\tilde{\chi}_{L} \otimes \tilde{\chi}_{R}$ transformations

$Z_{\tilde{A}} \partial_{\mu}\left\langle\tilde{J}_{\mu}^{A, i}(x) \hat{\mathscr{O}}(0)\right\rangle=\left[\bar{\eta}\left(\eta, g_{s}, \rho, \lambda_{0}\right)-\eta\right]\left\langle\left[\bar{Q}_{L}\left\{\frac{\tau^{i}}{2}, \Phi\right\} Q_{R}-\bar{Q}_{R}\left\{\frac{\tau^{i}}{2}, \Phi^{\dagger}\right\} Q_{L}\right](x) \hat{\mathscr{O}}(0)\right\rangle+\mathrm{O}\left(b^{2}\right)$,

where $\tilde{J}_{\mu}^{A, i}=\bar{Q}_{L} \gamma_{\mu} \gamma_{5} \frac{\tau^{i}}{2} Q_{L}$ is the axial current and $Z_{\tilde{A}}$ its renormalization constant. $\bar{\eta}$ is a $\mu_{0}^{2}$ independent dimensionless function that controls the mixing of the Wilson-like term with the Yukawa operator. Setting $\eta=\eta_{c r}\left(g_{s}^{2}, \rho, \lambda_{0}\right)$ such that $\eta_{c r}\left(g_{s}^{2}, \rho, \lambda_{0}\right)-\bar{\eta}\left(\eta_{c r} ; g_{s}^{2}, \rho, \lambda_{0}\right)=0$ the SDEs take 
the form of Ward-Takahashi identities (WTI) and the fermionic chiral transformations $\tilde{\chi}_{L} \times \tilde{\chi}_{R}$ become (approximate) symmetries [2] of the model (1.1).

The situation where $\hat{\mu}_{\phi}^{2}<0$ and $\mathscr{V}(\Phi)$ has a double-well shape with $\langle\Phi\rangle=v$, and the $\chi_{L} \times \chi_{R}$ symmetry is realized à la Nambu-Goldstone (NG) is physically more interesting. In this phase a non-perturbative term is expected/conjectured [1] to appear in the effective Lagrangian of the theory. Indeed the occurrence of the non-perturbative term can be interpreted by stipulating that the effective action contains the term $\Gamma \supset c_{1} \Lambda_{s}\left[\bar{Q}_{L} U Q_{R}+\mathrm{hc}\right]$, which provide a mass for the fermions. Here $\Lambda_{s}$ is the RGI scale of the theory and $U=\Phi / \sqrt{\Phi^{\dagger} \Phi}$ is a dimensionless effective field transforming as $U \rightarrow \Omega_{L} U \Omega_{R}^{\dagger}$ under $\chi_{L} \times \chi_{R}$, it represents the exponential Goldstone boson map and makes sense only if $v \neq 0$ [1], the latter term provide a mass for the fermions.

\section{Numerical investigation}

The arguments given in Ref. [1] imply that the NP mechanism leading to elementary fermion mass generation (if any) occurs even if virtual fermion effects are neglected, then in this first investigation we decided to work in the quenched fermion approximation, thus gauge and scalar configurations can be generated independently from each other. The details of the lattice regularization of (1.1) we use in our numerical study can be found in [3]. Due to the exact $\chi_{L} \times \chi_{R}$ symmetry the lattice artefacts are only $\mathrm{O}\left(b^{2 n}\right)$. In the present quenched study exceptional configurations of the gauge fields and the scalars can occur in the Monte Carlo sampling leading to small eigenvalues of lattice Dirac operator. In order to control exceptional configurations we add a twisted mass term in the action

$$
S_{\text {lat }}^{\text {toy }+ \text { tm }}=S_{\text {lat }}+i \mu b^{4} \sum_{x} \bar{\Psi} \gamma_{5} \tau_{3} \Psi
$$

at the price of introducing a soft (hence harmless) breaking of $\chi_{L, R}$ (and $\tilde{\chi}_{L, R}$ when restored). All the results will be extrapolated to $\mu \rightarrow 0$.

We simulate at three different values of the bare gauge coupling $\beta=5.75,5.85$ and 5.95, which correspond to three different lattice spacings $0.15,0.12$ and $0.10 \mathrm{fm}$, assuming for the Sommer scale fixed $r_{0}=0.5 \mathrm{fm}[4,5]$. The coefficient of the Wilson-like parameter in eq. (1.4) is kept fixed at $\rho=1.96$. In the NG phase for the three lattice spacing the parameters of the scalar sector are fixed by the renormalization conditions $M_{\sigma}^{2} r_{0}^{2}=1.284$ and $\lambda_{R} \equiv M_{\sigma}^{2} /\left(2 v_{R}^{2}\right)=0.441$ where $M_{\sigma}$ is the mass of the (non-Goldstone boson) scalar particle. We find that the values of the bare parameters that satisfy the renormalization condition are $\left(b m_{\phi}\right)^{2}=-0.5941,-0.5805,-0.5756$ and $\lambda_{0}=0.5807,0.5917,0.6022$ respectively. The phase in which the theory lives is selected by taking the bare scalar mass $m_{\phi}^{2}$ either larger (Wigner) or smaller (NG phase) than $m_{c r}^{2}$, where $m_{c r}^{2}$ is the value of $m_{\phi}^{2}$ at which the scalar susceptibility diverges in the infinite volume limit.

2.1 Wigner phase: To determine the critical value of the bare coupling $\eta$ at which the $\tilde{\chi}$ symmetry is restored we look, in the Wigner phase, for the value of $\eta$ at which

$$
r_{A W I}\left(\eta_{c r}, \rho\right)=\left.\frac{b^{6} \sum_{\mathbf{x}, \mathbf{y}}\left\langle P^{1}(0)\left[\partial_{0}^{F W} \tilde{A}_{0}^{1, B W}\right](x) \varphi_{0}(y)\right\rangle}{b^{6} \sum_{\mathbf{x}, \mathbf{y}}\left\langle P^{1}(0) \tilde{D}_{P}^{1}(x) \varphi_{0}(y)\right\rangle}\right|_{\eta=\eta_{c r}}=0, \quad \varphi_{0}=\frac{1}{2} \operatorname{Tr}[\Phi] .
$$


where $\tilde{A}_{\mu}^{i, B W}(x)=\frac{1}{2} \bar{Q}(x-\hat{\mu}) \gamma_{\mu} \gamma_{5} \frac{\tau^{i}}{2} U_{\mu}(x-\hat{\mu}) Q(x)+\frac{1}{2} \bar{Q}(x) \gamma_{\mu} \gamma_{5} \frac{\tau^{i}}{2} U_{\mu}^{\dagger}(x-\hat{\mu}) Q(x-\hat{\mu})$ is the axial current, and $P^{i}(x)=\bar{Q}(x) \gamma_{5} \frac{\tau^{i}}{2} Q(x), D_{P}^{i}=\bar{Q}_{L}\left\{\Phi, \frac{\tau^{i}}{2}\right\} Q_{R}-\bar{Q}_{R}\left\{\frac{\tau^{i}}{2}, \Phi^{\dagger}\right\} Q_{L}$. The time separation $y_{0}-x_{0}$ is kept fixed at $0.6 \mathrm{fm}$. The typical profile of $r_{A W I}(2.2)$ is shown in Fig. 1a. It exhibits two plateaux because different intermediate states dominates the three-point functions in the (2.2) . In Fig. $1 \mathrm{~b}$ we plot the extrapolation in $\eta$ of $r_{A W I}$ through $r_{A W I}=0$. All the values are taken from the time plateau region at $x_{0}$ smaller than $T / 2$ that we call region $\mathrm{A}$. We call region $\mathrm{B}$ the time plateau region of $r_{A W I}$ corresponds to $x_{0}$ larger than $T / 2$. The $r_{A W I}$ values extracted from the time region $\mathrm{B}$ are expected to differ from those coming from the time region $\mathrm{A}$ only due to lattice artefacts. In fact, we check in Fig. 1c that the difference in the values of $\eta_{c r}$ obtained by evaluating $r_{A W I}$ from the two plateaus of regions $\mathrm{A}$ and $\mathrm{B}$ vanishes as $\mathrm{O}\left(b^{2}\right)$ in the continuum limit.

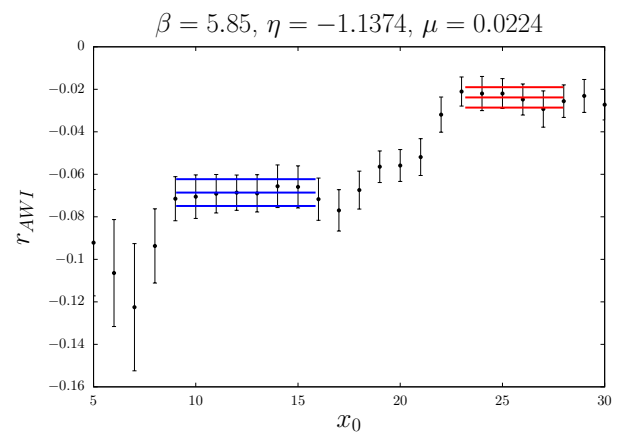

(a)

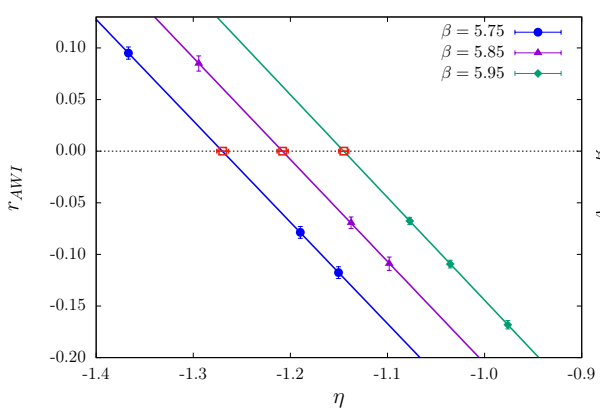

(b)

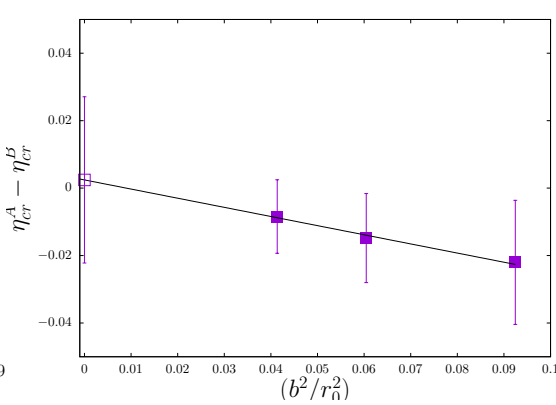

(c)

Figure 1: (a) typical plot of $r_{A W I}$ against $x_{0} / b$, here for the case of $\eta=-1.1374, b \mu=0.0224$ and $\beta=5.85$. The two time regions where $r_{A W I}$ shows a plateau are $A: x_{0} \sim[0.9,1.8] \mathrm{fm}$ and $B: x_{0} \sim[2.7,3.3] \mathrm{fm}$. (b) $r_{A W I}$ as a function of $\eta$ at $\beta=5.75,5.85$ and 5.95. The values of $\eta_{c r}$ at which $r_{A W I}=0$ are denoted by red squares and are $\eta=-1.271(10),-1.207(8)$ and $-1.145(6)$ respectively. (c) The difference $\eta_{c r}^{A}-\eta_{c r}^{B}$ vs. $b^{2} / r_{0}^{2}$, where $\eta_{c r}^{A(B)}$ is estimated by using the plateau value of $r_{A W I}$ in the time region $\mathrm{A}(\mathrm{B})$. The value of $\eta_{c r}^{A}-\eta_{c r}^{B}$ extrapolated to the continuum limit is well compatible with zero.

2.2 NG phase: to check whether the conjectured mass generation actually occurs we compute in the NG phase on a lattice of size $L=T / 2 \sim 2.4 \mathrm{fm}$ the effective quark PCAC mass as obtained from the axial Ward identity

$$
m_{A W I}^{R} \equiv \frac{Z_{\tilde{A}}}{Z_{P}} m_{A W I}=\left.\frac{Z_{\tilde{A}} \sum_{\mathbf{x}} \partial_{0}\left\langle\tilde{A}_{0}^{i}(x) P^{i}(0)\right\rangle}{2 Z_{\tilde{P}} \sum_{\mathbf{x}}\left\langle P^{i}(x) P^{i}(0)\right\rangle}\right|_{\eta_{c r}}, P^{i}=\bar{Q} \gamma_{5} \frac{\tau^{i}}{2} Q
$$

For practical reasons $Z_{P}^{-1}$ is evaluated in a hadronic scheme defined in the Wigner phase by taking $Z_{P}^{-1}=r_{0}^{2} G_{P S}^{W}=r_{0}^{2}\left\langle 0\left|P^{1}\right| \text { PS meson }\right\rangle^{W}$, while we can trade $Z_{\tilde{A}}$ with $Z_{\tilde{V}}$ due to $\chi_{L} \times \chi_{R}$ symmetry [3]. An another check that the effective PCAC mass $\frac{Z_{\tilde{A}}}{Z_{P}} m_{A W I}$ is non-zero thus indicates the presence in the effective lagrangian of a fermion mass term, is the fact of the mass of the lowest lying pseudoscalar meson state ( computed from the $\left\langle P^{1} P^{1}\right\rangle$ correlator) is not vanishing. The propagation of the uncertainty of $\eta_{c r}$ on all the observables gives the dominant contribution to the total error. 
Both the PCAC mass and $M_{P S}$ have been computed for several values of $\mu$ and $\eta$. These values have been extrapolated to $\mu \rightarrow 0$ and interpolated to $\eta=\eta_{c r}$. In order to have a control over the systematics in our simulation we have repeated our analysis modifying:

1. the plateau choice for $r_{A W I}$, which was taken in the time region either A or B of Fig. 1a;

2. the fit function for $r_{A W I}$ in the Wigner phase, which was chosen to be either of the form $K_{0}+K_{1} \eta+K_{2} \mu$ or extended by adding the non-linear term $K_{4} \mu^{2}$ (data are very well linear in $\eta$ ). In addition the average over the results from the two fits above was considered;

3. the choice of the time plateau for $M_{P S}$ and $m_{A W I}$, taken to be either $[1.4,2.2] \mathrm{fm}$ or $[1.1,2.4] \mathrm{fm}$;

4. the fit functions for $m_{A W I}$ and $M_{P S}^{2}$ in the NG phase, which were both either truncated to $Y_{0}+Y_{1} \eta+Y_{2} \mu$ or extended by adding in turn terms non-linear in $\eta$ and/or $\mu$, such as $Y_{4} \mu^{2}$, $Y_{3} \eta^{2}+Y_{4} \mu^{2}$ or $Y_{3} \eta^{2}+Y_{4} \mu^{2}+Y_{5} \eta \mu$.

For each combination of the above choices $r_{0} M_{P S}$ and $2 r_{0} m_{A W I} Z_{\tilde{V}} Z_{P}^{-1}$ were extrapolated linearly in $b^{2} / r_{0}^{2}$ to the continuum limit. To estimate the impact of possible $\mathrm{O}\left(b^{4}\right)$ and higher order lattice artifacts, we have also considered a continuum extrapolation excluding the coarsest lattice spacing, but imposing that the two choices of taking either $\eta_{c r}^{A}$ or $\eta_{c r}^{B}$ yield the same continuum value. For a typical choice of the time plateau and the fit function in $\eta$ and $\mu$ for $M_{P S} r_{0}$, in Fig. 2a we show the continuum limit extrapolations obtained by working at $\eta_{c r}^{A}$ or at $\eta_{c r}^{B}$ and using data at three lattice spacings, as well as the combined continuum extrapolation that results from using data on the two finer lattices at both $\eta_{c r}^{A}$ and $\eta_{c r}^{B}$. The analogous comparison for the renormalized ratio $2 r_{0} m_{A W I} Z_{\tilde{V}} Z_{P}^{-1}$ is presented in Fig. $2 \mathrm{~b}$.

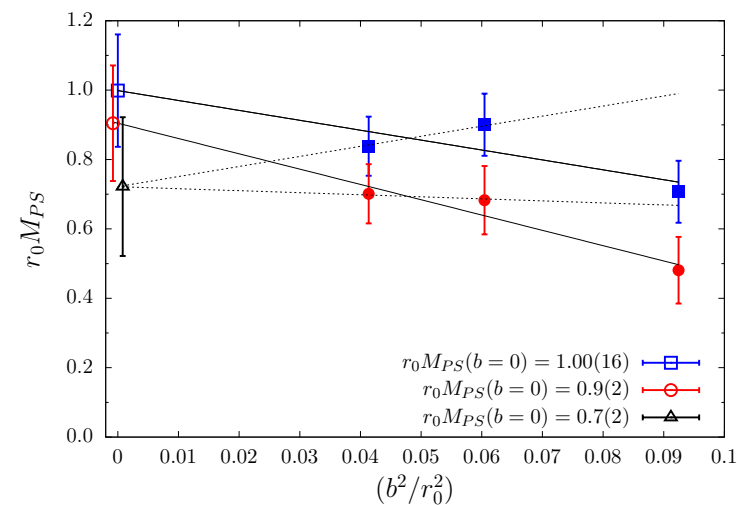

(a)

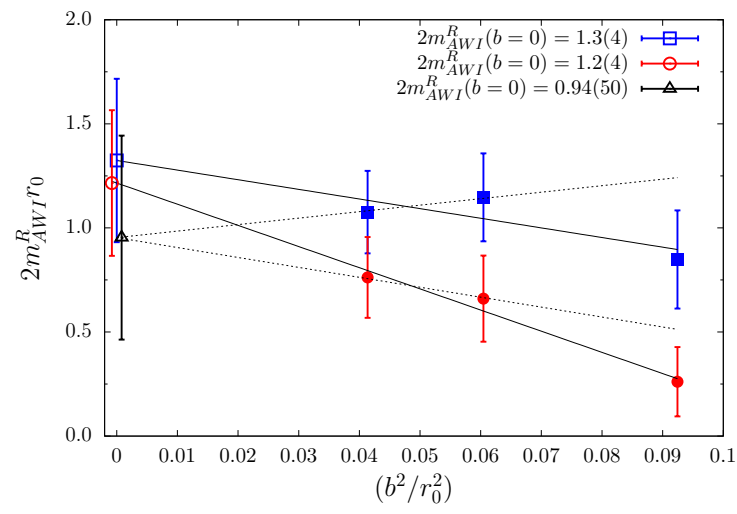

(b)

Figure 2: (a) Continuum limit extrapolations of $M_{P S} r_{0}$ data as obtained by working at $\eta_{c r}^{A}$ (blue squares) or at $\eta_{c r}^{B}$ (red circles) and using data at three lattice spacings as well as combining data on the two finer lattice at both $\eta_{c r}^{A}$ and $\eta_{c r}^{B}$ under the constraint of a common continuum limit. A conservative choice of the time plateau for $M_{P S}$ and the fit ansatz $M_{P S}^{2}=Y_{0}+Y_{1} \eta+Y_{2} \mu+$ $Y_{3} \eta^{2}+Y_{4} \mu^{2}+Y_{5} \eta \mu$ were adopted. (b) Data and continuum limit extrapolations of $2 m_{A W I}^{R} r_{0} \equiv$ $2 r_{0} m_{A W I} Z_{\tilde{V}} Z_{P}^{-1}$ for the same parameters choice as in (a). The fit ansatz $m_{A W I}=Y_{0}+Y_{1} \eta+Y_{2} \mu+$ $Y_{3} \mu^{2}$ was adopted. 
The combination of the analysis variants discussed above produced 72 different analyses, none of which gave a result for $m_{A W I}$ or $M_{P S}$ that is compatible with zero (i.e. with the no mechanism scenario). The median over all the analyses, excluding only a few cases where $M_{P S}^{2}$ yields a $\chi^{2}$ value per degree of freedom larger than 2 , gives

$$
r_{0} M_{P S}=0.93 \pm 0.09 \pm 0.10 \rightarrow 0.93(14), \quad r_{0} m_{A W I}^{R}=1.20 \pm 0.39 \pm 0.19 \rightarrow 1.20(43)
$$

where the first error is statistical and the second systematic while the error in bracket is their sum in quadrature. Error were estimated by assigning equal weight to all analyses, following the method of Ref. [6].

Further strong evidence for a non-zero fermion mass in the EL comes from the following "reductio ad absurdum" argument. Let us assume that no fermion mass term is generated. If this were the case, at $\eta=\eta_{c r}$ one should have $M_{P S}=\mathrm{O}\left(b^{2}\right)$, and hence $M_{P S}^{2}$ would approach a vanishing continuum limit with only $\mathrm{O}\left(b^{4}\right)$ artifacts. In Fig. 3 we plot $r_{0}^{2} M_{P S}^{2}$ as a function of $\left(b / r_{0}\right)^{4}$ working

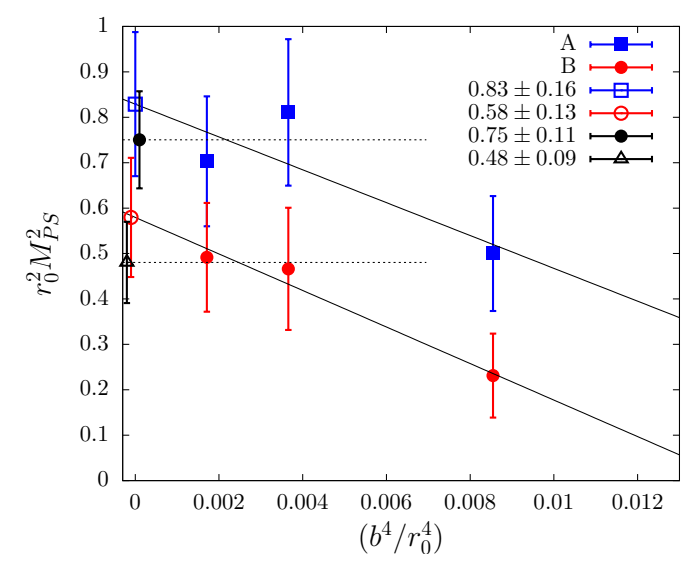

Figure 3: $r_{0}^{2} M_{P S}^{2}$ against $\left(b / r_{0}\right)^{4}$ in the NG phase: full (dotted) straight lines show the continuum extrapolation linear in $b^{4}$ using data at all three (only the two finest) lattice spacings. Blue square are the values computed at $\eta_{c r}^{A}$ while the red circle are the values at $\eta_{c r}^{B}$

at $\eta_{c r}^{A}$ and $\eta_{c r}^{B}$. On a $\left(b / r_{0}\right)^{4}$ scale the lattice results for $r_{0}^{2} M_{P S}^{2}$ lie very close to the continuum limit, which appears to be non-zero by more than five standard deviations. Thus the hypothesis of "no mechanism" is not supported by lattice data.

2.3 Numerical investigation at larger values of $\rho$ : Having found non vanishing values of $m_{A W I}$ and $M_{P S}$ in the continuum limit at $\eta_{c r}$ in the NG phase, as conjectured in Ref. [1], we check the behaviour of this masses as a function of $\rho$. In Ref. [1] was conjectured that $m_{A W I}$ and $M_{P S}^{2}$ are increasing functions of $\rho$ and for small $\rho$ they should increase at least as $\mathrm{O}\left(\rho^{2}\right)$. In order to check the $\rho$ dependence of $m_{A W I}$ and $M_{P S}^{2}$ we have thus studied the case of a 1.5 times larger $\rho$ value, namely $\rho=2.94$, for the intermediate lattice spacing ( $\beta=5.85, a \sim 0.123 \mathrm{fm}$ ) while keeping the scalar sector parameters fixed to the values given in $\S 2$. In the Wigner phase, we found that changing $\rho$ from 1.96 to 2.94 leads to a substantial change of $\eta_{c r}$, from $-1.207(8)$ to $-1.838(13)$. In the NG phase we observe as expected a significant increase of $2 r_{0} m_{A W I} Z_{\tilde{A}} Z_{P}^{-1}$ from 1.5(0.21) to 2.80(0.36) Fig. 4b, as well as a similar increase of $M_{P S}^{2} r_{0}^{2}$ Fig. 4a. 


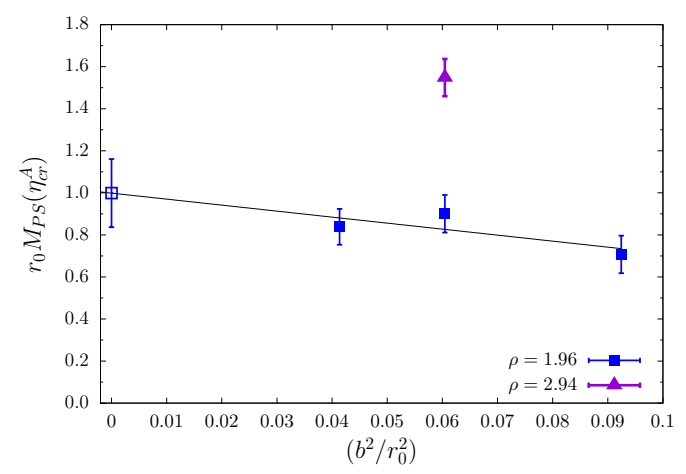

(a)

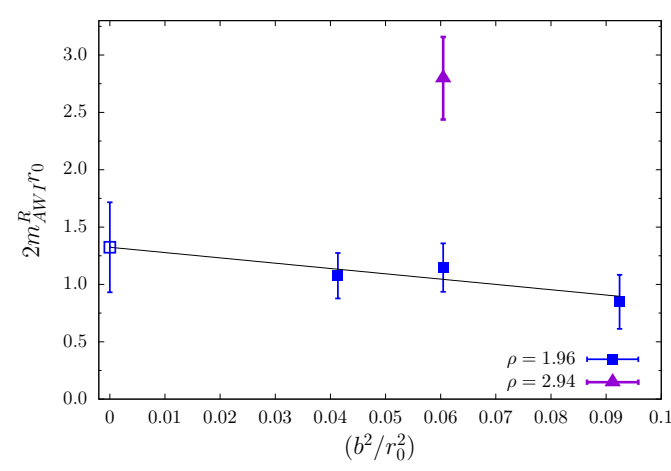

(b)

Figure 4: (a) Data and continuum limit extrapolations of $M_{P S} r_{0}$ as obtained in the NG phase at $\eta_{c r}^{A}$. A conservative choice of the time plateau for $M_{P S}$ and the fit ansatz $M_{P S}^{2}=Y_{0}+Y_{1} \eta+Y_{2} \mu+$ $Y_{3} \eta^{2}+Y_{4} \mu^{2}+Y_{5} \eta \mu$ were adopted. Blue squares refer to $\rho=1.96$ and the dark violet triangle to $\rho=2.94$. (b) Data and continuum limit extrapolations of $2 m_{A W I}^{R} r_{0} \equiv 2 r_{0} m_{A W I} Z_{\tilde{V}} Z_{P}^{-1}$ obtained at $\eta_{c r}^{A}$ in the NG phase. The fit ansatz $m_{A W I}=Y_{0}+Y_{1} \eta+Y_{2} \mu+Y_{3} \mu^{2}$ was adopted.

\section{Conclusions}

In this contribution we illustrate our numerical study of the toy model (1.1). Our results are compatible with the conjecture [1] of a new non-perturbative (NP) mechanism for elementary mass generation. Once the bare parameters are chosen so as to ensure maximal restoration of fermion chiral symmetries and be in the spontaneously broken phase of the $\chi_{L} \times \chi_{R}$ symmetry, a renormalization group invariant fermion mass of the order of the $\Lambda_{s}$ parameter is generated. This result establishes the occurrence of a non-perturbative obstruction ("anomaly") to the recovery of broken fermionic chiral symmetries, which is responsible for the dynamically generated fermion mass term. The latter, being unrelated to the Yukawa operator, is expected to become independent of the scalar field expectation value $(v)$ in the limit $\Lambda_{s} / v \rightarrow 0$. Extension of this mechanism to weak interactions and possible phenomenological implications are discussed in the contribution of R. Frezzotti [7]

\section{References}

[1] R. Frezzotti and G. C. Rossi, Phys. Rev. D92 (2015) 054505.

[2] M. Bochicchio, L. Maiani, G. Martinelli, G. C. Rossi and M. Testa, Nucl. Phys. B 262 (1985) 331.

[3] S. Capitani et al., EPJ Web Conf. 175 (2018) 08009.

[4] ALPHA collaboration, M. Guagnelli, R. Sommer and H. Wittig, Nucl. Phys. B535 (1998) 389.

[5] S. Necco and R. Sommer, Nucl. Phys. B622 (2002) 328.

[6] N. Carrasco et al., Nuclear Physics B 887 (2014) 19.

[7] R. Frezzotti and G. C. Rossi, PoS, 36th International Symposium on Lattice Field Theory (Lattice 2018) East Lansing, MI, United States, July 22-28, 2018. 